\title{
Die operative Behandlung von Frakturen im Bereich der Brust-/Lendenwirbelsäule
}

\author{
Hans-Jörg Oestern
}

\section{Zusammenfassung}

Frakturen des thoraco-lumbalen Wirbelsäulenabschnittes machen mehr als 50\% aller Wirbelfrakturen aus. Die genaue Analyse der Röntgenaufnahmen erlaubt bereits eine Klassifizierung der Fraktur, die durch die Computertomographie ergänzt wird. Etwa $1 / 3$ der Patienten mit Wirbelfrakturen im thorako-lumbalen Bereich weisen Zusatzverletzungen auf. Wir unterscheiden dorsale und ventrale Stabilisierungsverfahren.
Dorsal werden winkelstabile Implantate angewandt, die zusätzlich noch eine Repositionsmöglichkeit erlauben. Die Fehlplatzierung der Schanzschen Schrauben liegt in der Literatur bei ca. 6,1\%. Die ventralen Verfahren erlauben Stabilisierung und Dekompression über einen Zugang. Die früher gebräuchlichen, größeren Zugänge sind durch die laterale Thorakotomie oder thoraskopische Verfahren mit endoskopischem Zwerchfellsplitting ergänzt worden. Ventrale Abstützung erfolgt durch Span auch in Kombination mit einer Platte und durch cages.

\section{Einleitung}

Den thorakolumbalen Übergang (Th11 bis L2) betreffen mehr als $50 \%$ aller Wirbelfrakturen. Ursächlich verantwortlich ist zum einen die fehlende stabilisierende Wirkung des Brustkorbes in diesem Bereich, zum anderen ist der Übergang zwischen der Kyphose der Brustwirbelsäule und der Lordose der Lendenwirbelsäule biomechanisch gefährdet. Auch die Lage der kleinen Wirbelgelenke mit der Änderung von einem frontalen Verlauf im thorakalen zu einem sagittalen im lumbalen Bereich lässt die Rotationssteifigkeit erheblich ansteigen und erhöht die Verletzungsgefahr. Auf der anderen Seite wird die Flexions- und Extensionsbeweglichkeit in diesem Wirbelsäulenabschnitt durch die Stellung der Gelenkflächen erhöht.

OP-JOURNAL 2001; 17: 150-155

(c) Georg Thieme Verlag Stuttgart . New York

\section{Diagnostik}

1. Röntgenaufnahmen erlauben bei genauer Interpretation eine Aussage über den Instabilitätsgrad. Das Computertomogramm ist eine unverzichtbare Ergänzung jeder Untersuchung der instabilen Wirbelsäule.

2. Die neurologischen Zusatzverletzungen nehmen vom Typ A zum Typ C zu. Frakturen der Brustwirbelsäule haben zu über 40 \% Begleitverletzungen.

Der thorakolumbale Übergang wird auf konventionellen Röntgenaufnahmen der BWS und LWS häufig nur randständig abgebildet. Diagnostisch muss auf folgende Veränderungen geachtet werden: Seitversetzung von Dornfortsätzen und Wirbelkörpern, senkrechter und querer interpedikulärer Abstand, seitliche Höhenminderung von Wirbelkörpern, unterschiedliche Abstände zwischen Dornfortsätzen, Asymmetrie vorderer und hinterer Wirbelelemente als Zeichen der axialen Rotation.

Auf der seitlichen Aufnahme kann das Ausmaß einer Wirbelkörperkompression ebenso wie die Höhe des Intervertebral- raumes und die Verlagerung von Hinterkantenfragmenten bestimmt werden.

Die Computertomographie ist unverzichtbare Ergänzung jeder Untersuchung der verletzten Wirbelsäule. Besonders gut können der Spinalkanal und seine mögliche Einengung dargestellt werden.

\section{Einfluss der Spinalkanaleinengung auf neurologische Ausfallserscheinungen}

Nach Denis [11] bedeutet eine Einengung von mehr als $75 \%$ des Spinalkanalquerschnittes neurologische Ausfälle (Frankel B für den Konusbereich und Frankel C für den Cauda-equina-Bereich). Hashimoto et al. [16] berichteten bei Berstungsbrüchen über eine neurologische Beteiligung auf Höhe von Th 11 bis Th12 mit 35\%iger und größerer Einengung, auf Höhe L1 mit 45\% oder mehr und bei L2 und darunter mit 55\% und mehr. Diese Ergebnisse wurden bestätigt durch Kim [20]. Ebenso steigt die Häufigkeit einer neurologischen Zusatzverletzung vom Typ A zum Typ C. Während bei den A-Verletzungen in 22\% neurologische Störungen beobachtet wurden, lag dieser Prozentsatz bei den B-Verletzungen schon bei $28 \%$ und bei den C-Verletzungen bei 51\% [22].

\section{Zusatzverletzungen}

Meyer [25] fand bei $41 \%$ von 376 Patienten mit BWS-Verletzungen gleichzeitig ein Thoraxtrauma. Dieser hohe Prozentsatz hängt damit zusammen, dass ein Teil der axial einwirkenden Kräfte während des Unfalls auf den Brustkorb übertragen wird. Die Steifigkeit der BWS nimmt durch den Rippenkorb um das 2,5- bis 4 fache zu. Typischerweise treten folgende Begleitverletzungen auf: 1. Rippenserienfrakturen, besonders zu beachten sind die 1 . und 2. Rippe, die etwa bei $10 \%$ aller Patienten mit Verletzungen der BWS gebrochen sind. 2. Sternumfrakturen, 3. Hämatopneumothorax, 4. Claviculafrakturen und 5. Scapulafrakturen, 
6. Mediastinalverbreiterung, 7. Gefäßverletzungen.

\section{Zusatzverletzungen bei Frakturen der LWS}

Im Falle von Distraktionsläsionen kann es zu Verletzungen von Oberbauchorganen kommen (z.B. gedeckte Dünndarmruptur), auch Verletzungen der Nieren und ableitenden Harnwege sind beschrieben.

In der Sammelstudie der Deutschen Gesellschaft für Unfallchirurgie hatten unter den 682 Patienten 35,9\% eine oder mehrere Begleitverletzungen und 42 (6,2\%) waren polytraumatisiert. Unter den Patienten mit Begleitverletzungen hatten 62,7\% Extremitäten- und 15,7\% Beckenverletzungen, bei $7 \%$ lagen abdominelle und bei $34,1 \%$ thorakale Verletzungen vor. Ein Schädelhirntrauma hatten $25,1 \%$ erlitten [22].

\section{Behandlungsziele}

Lorenz Böhler [6] hat sehr treffend die Behandlungsziele formuliert: Wir wollen nicht nur, dass die Lähmung verschwindet, sondern dass der Verletzte auch seine frühere normale Form, Beweglichkeit und Kraft wiedererlangt. Die Stabilität der Verletzung und neurologische $\mathrm{Zu}$ satzverletzungen bestimmen die Indikation zum konservativen oder operativen Vorgehen. Als stabil können Verletzungen nur dann gelten, wenn keine Reposition erforderlich ist bzw. unter physiologischen Belastungen keine weitere Verformung eintritt [5]. Einzurichtende Läsionen sind immer als instabil zu betrachten, sie müssen zur Erhaltung des Repositionsergebnisses retiniert bleiben. Demnach können nur Kompressionsverletzungen des Typs A 1.1 bis A 1.3 sowie einfache, unverschobene Spaltbrüche (A 2.1 und A 2.2) als stabil gelten.

\section{Heilungsvorgänge}

Die einzelnen Anteile des Bewegungssegmentes weisen ein unterschiedliches Heilungsverhalten auf:

Ein zerrissener dorsaler Ligamentkomplex verheilt unter Ausbildung einer Narbe mit fehlender zuggurtender Funktion des hinteren Kapselbandapparates, so dass eine zunehmende Kyphosierung des Bewegungssegmentes mit Verlängerung der interspinösen Distanz und Fehlbelastung der kleinen Wirbelgelenke resultiert. Die eingeschränkte Funktionsfähigkeit der dorsalen Bänder wird jedoch nur dann zu einem Problem, wenn die abstützende Funktion der vorderen Säule fehlt oder gestört ist [5].

Diskusverletzungen heilen nicht und führen im besten Fall zu einer ventralen Spangenbildung.

Die Brüche dorsaler Wirbelbogenanteile heilen dagegen ebenso wie Frakturen des Wirbelkörpers aus, so lange die Fragmente miteinander in Verbindung stehen.

\section{Operative Stabilisierungsverfahren Dorsale Verfahren}

Wir unterscheiden dorsale und ventrale Stabilisierungsverfahren. Die früher gebräuchlichen Drahtzuggurtungen und Verplattungen von Dornfortsätzen, Harrington-Kompressionsinstrumentarien und Kerbenplatten der AO haben nur noch historische Bedeutung und sind von winkelstabilen Implantaten wie dem Fixateur interne abgelöst worden. Den Fixateur interne gibt es mittlerweile in zahlreichen verschiedenen Ausführungen, er erlaubt an der Wirbelsäule alle notwendigen Repositions- und Stabilisierungsmöglichkeiten: Neutralisation, Distraktion und Zuggurtung. Das USS (Universal spine System) vereint 3- oder 4Punkt-Abstützungen mit kurzstreckiger, winkelstabiler Montage. Dazu können auch sagittale Verschiebungen und Rotationsfehlstellungen auf einfache Weise beseitigt werden.

\section{Ventrale Stabilisierungsverfahren}

Operationsverfahren von ventral bieten auf den ersten Blick große Vorteile $[4,12,18]$.

1. Man befindet sich am Hauptort einer Verletzung, die Zerstörung der vorderen Säule bestimmt in hohem Maße die weitere Prognose [21].

2. Die dorsale Muskulatur bleibt intakt.

3. Dekompression und Abstützung können über einen Zugang kombiniert werden.

Die Nachteile sind, dass sich das alleinige ventrale Verfahren aus biomechanischen Gründen nur für Typ-A-Verletzungen eignet. Aber auch bei diesen Läsionen werden im thorakolumbalen und lumbalen Bereich immer wieder Korrekturverluste, vor allem auch in der Frontalebene, beobachtet. Es entsteht bei mehrsegmentalen Fusionen nicht selten eine linkskonvexe Skoliose. Daneben sind Arrosionsblutungen großer Gefäße ebenso möglich, Revi- sionseingriffe sind risikoreicher und schwieriger [5].

\section{Beeinflussung neurologischer Symptome durch Entlastung}

Verlagerte Hinterkantenfragmente können teilweise resorbiert werden.

Die ausgesprochen seltene Rückenmarkszerreißung lässt sich ebenso wie die Folgen einer spinalen Ischämie nur sehr eingeschränkt beeinflussen. Eindeutig konnte, zumindest tierexperimentell, der Nachweis erbracht werden, dass die Krafteinwirkung und die Kraftdauer mit entsprechender Kompression des Rückenmarkes Ausmaß und Erholungsfähigkeit des Rückenmarkes bestimmen $[27,9]$. Dagegen konnte weder für inkomplette noch für komplette Querschnittsyndrome ein eindeutiger Nachweis der Überlegenheit einer operativen Dekompression gegenüber einer konservativen Behandlung allein durch Lagerung erbracht werden $[2,7,8]$. Bei der Bewertung der Zusammenhänge unter Zuhilfenahme des Manabe-Scores konnte Gertzbein [15] in einer Multizenterstudie zwar signifikant bessere Ergebnisse nach operativer Behandlung feststellen, bei Anwendung des Frankel-Scores traf das jedoch nicht mehr zu. Boerger [7] et al. untersuchten 275 Publikationen über die Behandlung von Berstungsbrücken. 60 erfüllten die minimalen Einflusskriterien und wurden näher analysiert. Nur 3 Veröffentlichungen waren prospektive Studien, die restlichen retrospektive/deskriptive Analysen. Keine der 60 Artikel schloss eine Kontrollgruppe ein. Aufgrund dieser Untersuchungen ließen sich keine signifikanten Verbesserungen der neurologischen Situation durch operative Verfahren nachweisen.

Durch serielle computertomographische Untersuchungen konnte nachgewiesen werden, dass verlagerte Hinterkantenfragmente sowohl nach operativer als auch nach konservativer Behandlung teilweise oder ganz resorbiert werden konnten. Mumford et al. [26] stellten eine signifikante Abnahme der Spinalkanaleinengung bei 41 Patienten von ursprünglich $37 \%$ ( 16 bis $66 \%$ ) auf $15 \%$ ( 3 bis $40 \%$ ) fest. Unter Umständen sind Pulsationen der paarigen longitudinalen venösen Plexus für diese Resorptionsvorgänge verantwortlich.

Da aufgrund des spinalen Schocks in den ersten Tagen keine genaue Aussage über 

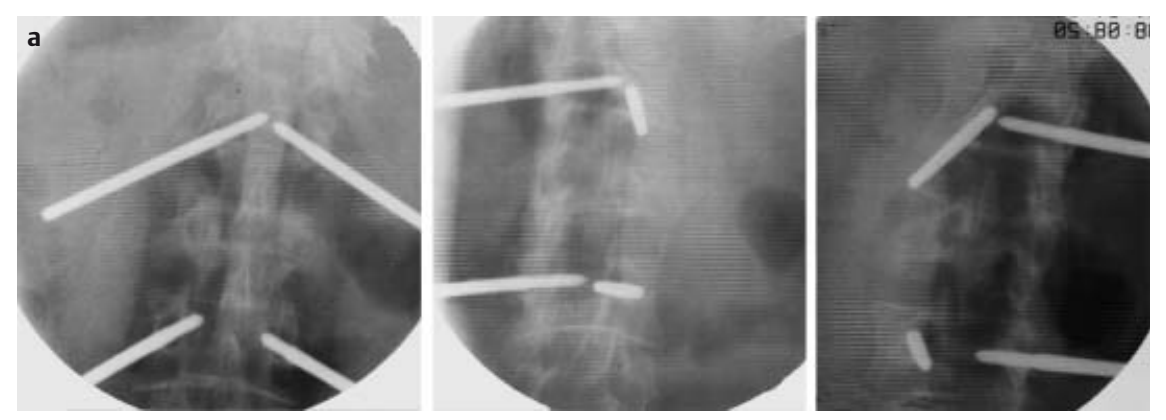

Abb.1 Platzieren der Kirschner-Drähte und Kontrollieren der Lage in beiden Ebenen, sowie Schrägaufnahmen in der a.p. Ebene (a). Austauschen gegen die Schanzschen Schrauben und Vorbiegen der USS-Stäbe (b). Zur Korrektur des Höhenverlustes bei frakturierter Hinterwand Einbringen des Halbringes, dosierte Distraktion mit der USS-Spreizzange (c). Anschließend Quer-Stabilisierung (d).
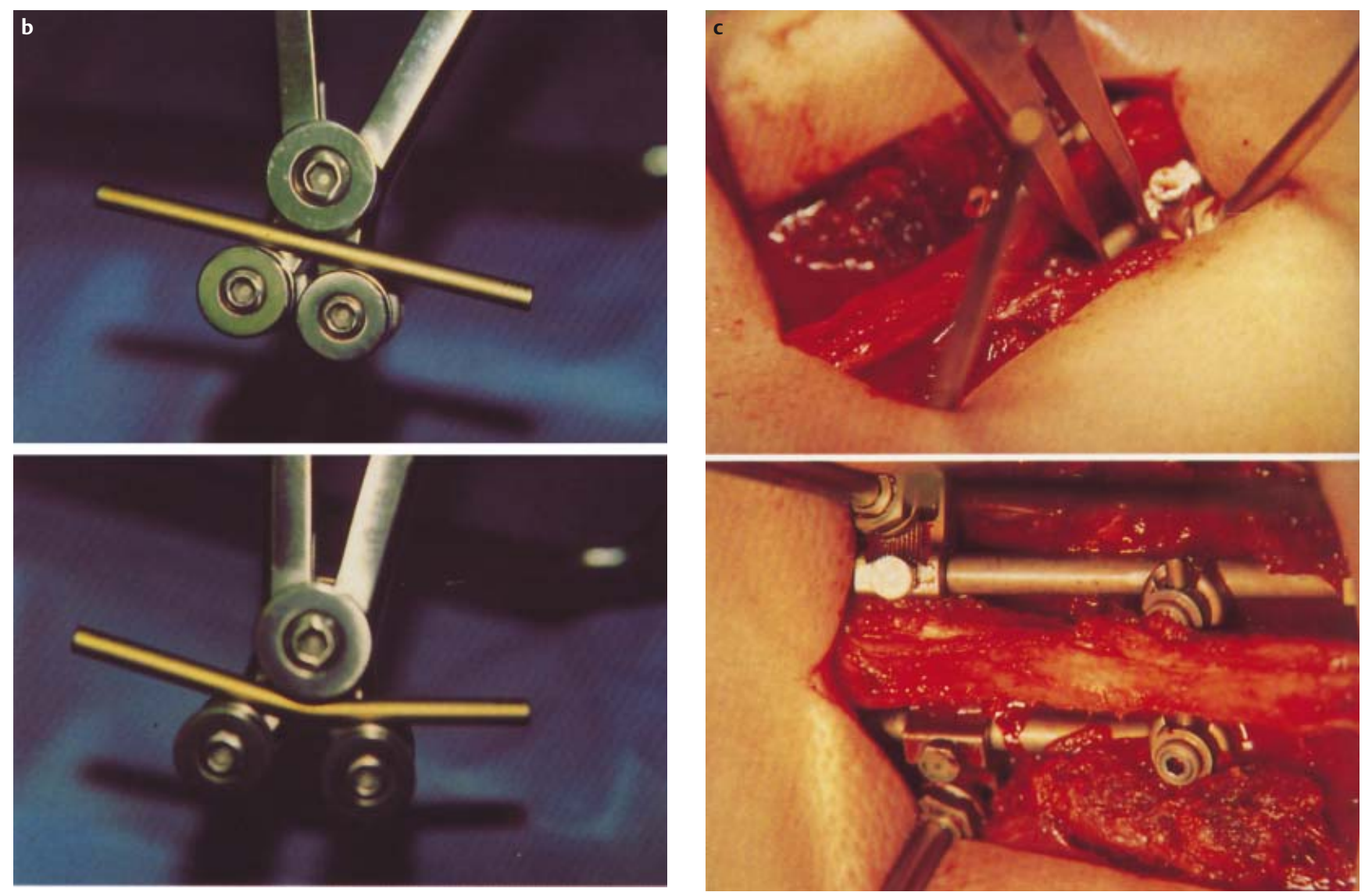

die Irreversibilität einer Läsion bestimmt werden kann, sollte auch eine komplette Querschnittslähmung mit entsprechendem Korrelat so schnell wie möglich reponiert und stabilisiert werden.

\section{Repositionstechnik}

Wichtigste Erstmaßnahme bei starker Höhenminderung oder Keilform ist zunächst die geschlossene Reposition, die unter Längszug an Armen und Beinen und anschließend durch einfache lordosierende Lagerung erreicht wird. Das Repositionsergebnis kann im seitlichen Bildwandlerröntgenbild dokumentiert werden. Für die Einrichtung eines Wirbelkörpers spielen die Nachbarstrukturen, also das vordere und hintere Längs- band, sowie vor allem der Anulus fibrosus die Hauptrolle. Möglicherweise entsteht durch den Längszug auch ein gewisser Unterdruck, der durch eine Sogwirkung für die Reposition Bedeutung besitzt.

\section{Dorsale Operationstechnik (Abb.1)}

Zur Präparation der Eintrittsstellen für die Schanzschen Schrauben wird am häufigsten ein Rongeur benutzt. Das transpedikuläre Bohrloch wird meistens mit einem Kirschen-Draht vorbereitet.

Der Eingriff erfolgt in Bauchlage. Ein Längszug zur geschlossenen Reposition und Retention kann durch Gewichte von 5 bis $10 \mathrm{~kg}$ an beiden Beinen verstärkt werden. Der Längszug lässt sich recht günstig, z.B. über die gepolsterten Fußhalter des Extensionstisches, applizieren. Zweckmäßigerweise wird über den seitlich eingebrachten Bildwandler die Höhe des verletzten Wirbels eingezeichnet. Die Fascia thoracolumbalis und die Fascie des M. erector trunci werden neben den Dornfortsätzen längs inzidiert. Die Muskelansätze werden zur Vermeidung von Blutungen mit dem Elektrokauter oder Skalpell und dann mit einem breiten Raspatorium abgeschoben, bis die Querfortsätze tastbar sind [19]. Die anatomische Landmarke zur Bestimmung des Pedikeleintrittpunktes kann sich zum einen aus der seitlichen Tangente am lateralen Rand des oberen Gelenkfortsatzes und einer weiteren senk- 

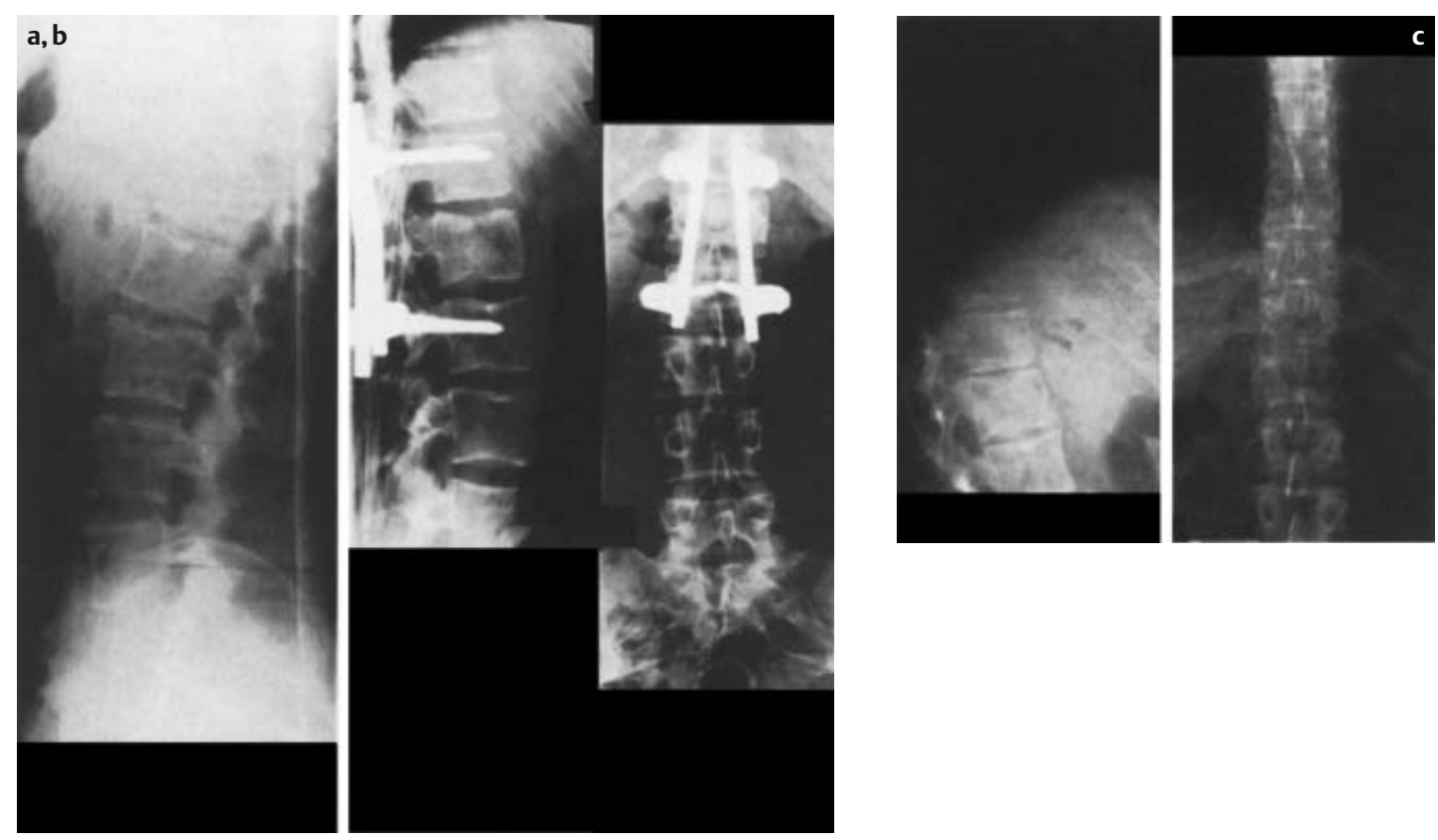

Abb. 2 Dorsale Instrumentierung bei LWK-1-Fraktur.

(a) Unfallbild,

(b) postoperatives

Ergebnis, (c) prä und postoperatives $\mathrm{CT}$,

(d) Zustand nach Metallentfernung Nachuntersuchungsbild 3 Jahre nach Unfall.

rechten Tangente entlang der lateralen Begrenzung der Lamina ergeben, wobei die Mitte zwischen diesen beiden Linien den Eintrittspunkt anzeigt. Eine weitere Möglichkeit besteht in der Bestimmung des Schnittpunktes der senkrechten Tangente am lateralen Rand des oberen $\mathrm{Ge}$ lenkfortsatzes und einer horizontalen Linie durch die Mitte des Processus transversus. Mit Hilfe von Kirschner-Drähten werden die Eintrittspunkte in beiden Ebenen markiert. Schwenken des orthograd eingestellten Bildwandlers in einem Winkel von jeweils $30^{\circ}$ zeigt die korrekte Platzierung der Insertionsstelle. Der Eintrittspunkt kann nun nach Präparation mit dem Rongeur mit einer winkelgetriebenen Bohrmaschine oder mit dem abgerundeten Teil eines Krischner-Drahtes unter leichten Hammerschlägen weiter eröffnet werden bis der Wirbelkörper erreicht ist. Es erfolgt dann nochmalige Kontrolle der eingebrachten KirschnerDrähte. Kommt es beim Einbringen zu einem Widerstand, muss eine leichte Richtungsänderung erfolgen. Nach entsprechender Kontrolle der Drähte werden diese nun durch Schanzsche Schrauben ersetzt, die möglichst in der deckplattennahen Spongiosa verankert werden sollten, da sie hier den besten Halt finden. Konvergierende Schrauben können länger gewählt werden, außerdem ist die Schraubenlage stabiler gegenüber seitlicher Abscherung. Die Längsträger werden nun mit Klemmbacken versehen, die über die Schanzschen Schrauben geführt werden, dabei ist ein Vorbiegen
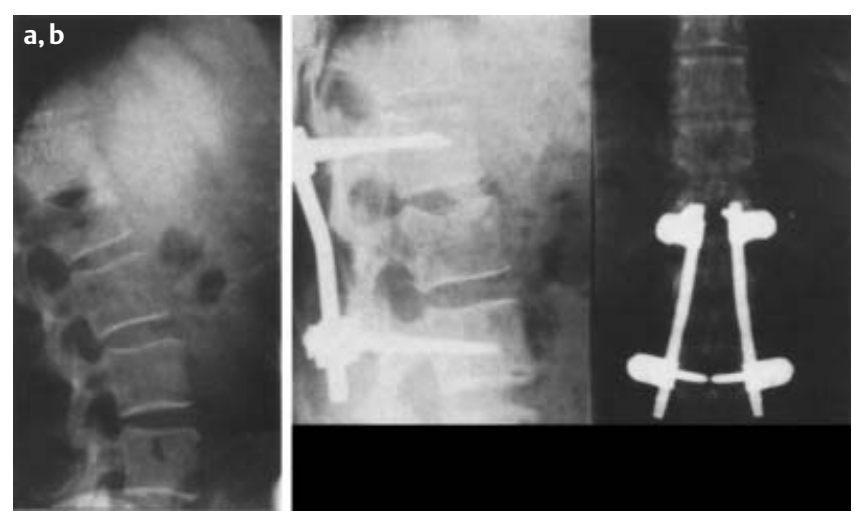

Abb. 3 Dorsale Instrumentierung bei BWK-12-Fraktur.

(a) Unfallbild,

(b) Röntgenverlauf 3 Monate nach Unfall.

im thorakolumbalen Bereich sinnvoll [3,17], (Abb. 2,3).

In einer Untersuchung von Bastian [1] an 31 Wirbelsäulenzentren in Deutschland über die Operationstechnik ergaben sich sehr viele Variationen. Die scharfe Dissektion der Muskulatur erfolgte am häufigsten mit dem Skalpell [21,31], unter Schonung der Muskelinsertion am Processus transversus und des dorsalen Astes der Segmentarterie. Am häufigsten wird zur Präparation der Pedikeleintrittsstelle ein Rongeur benutzt [22,31]. Das transpedikuläre Bohrloch wird meistenteils mit einem Kirschner-Draht vorbereitet $[11,31]$.

Daniaux [10] führte die Technik der transpedikulären Spongiosaplastik zur Wiederherstellung der vorderen Säule ein. Während Daniaux über recht gute Ergebnisse berichtete, konnte dies durch andere Arbeitsgruppen nicht immer bestätigt werden [21].

\section{Dorsale Dekompression}

Im lumbalen Bereich kann der Spinalkanal über eine Flavektomie eröffnet werden, im Cauda-equina-Bereich gestattet eine Laminektomie die Darstellung von Dura und Nervenwurzeln, ebenso lassen sich Knochenfragmente direkt mit dem Rongeur entfernen.

\section{Fehler und Gefahren der transpedikulären \\ Schraubenimplantation}

Bei einer Perforation der Pedikelkortikalis fanden George et al [14] 11\% weniger Halt bei Schraubenauszugstests. Bei einer zu ventralen Schraubenlage kann es zu Gefäßläsionen kommen, während bei lateraler Fehllage eine schlechte Festigkeit 

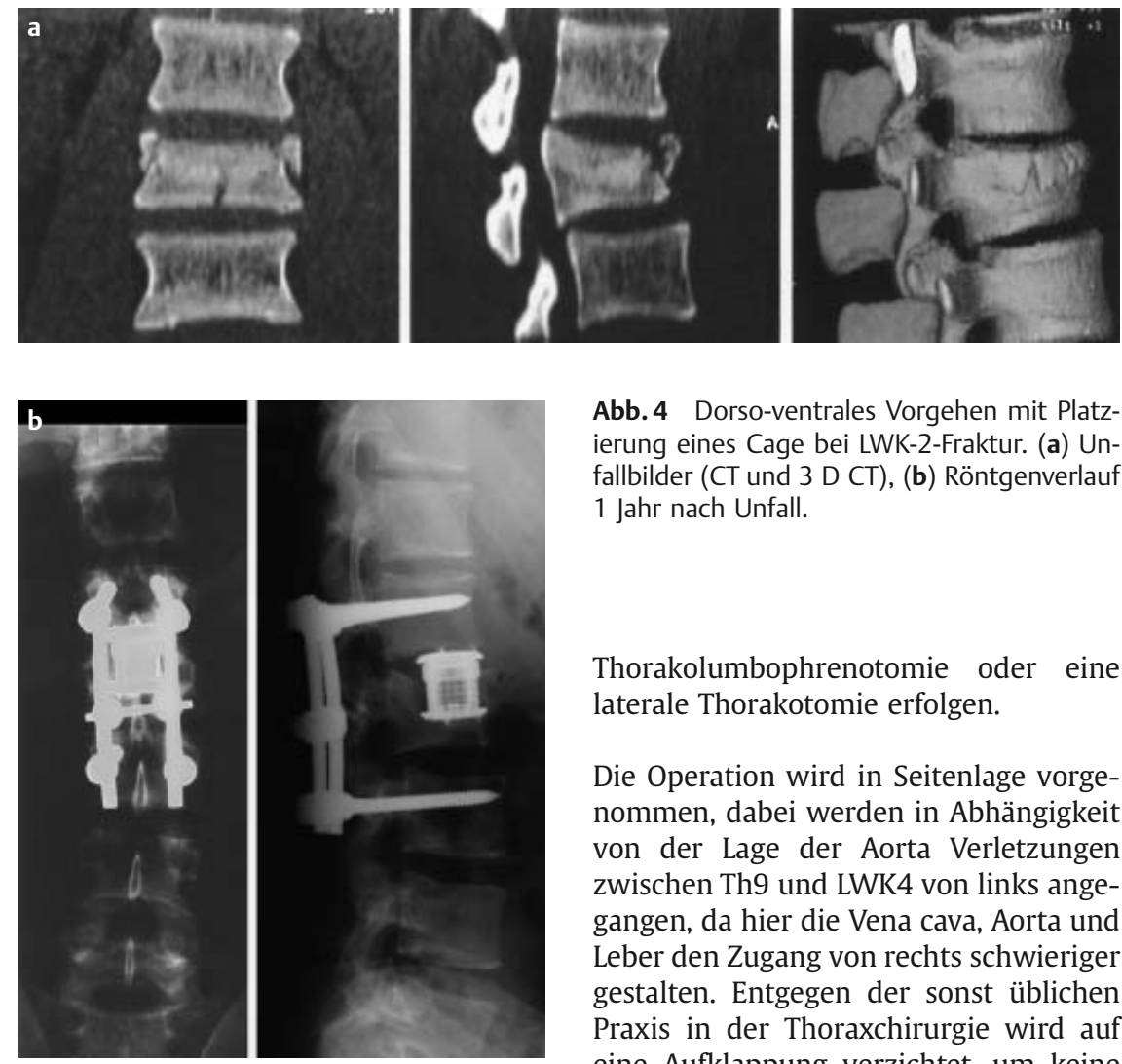

resultiert. Bei medialer Fehllage besteht nach Roy Camille [28] ein Spielraum von $2 \mathrm{~mm}$ im epiduralen Raum der Wirbelsäule, bei kaudaler Perforation des Pedikels kann es zu einer Verletzung der Nervenwurzel und bei kranialer zu einer Irritation der Bandscheibe kommen [13]. In der Sammelstudie der Deutschen Gesellschaft für Unfallchirurgie wurde bei 16,5\% der Patienten mindestens eine Schraubenlage als Fehllage gewertet. Dabei handelte es sich in mehr als der Hälfte um mediale Fehllagen. Insgesamt betrug die Rate von Schraubenfehllagen 6,1\% (139 von 2264) - [23].

\section{Ventrale Verfahren Operationstechnik}

Zur ventralen Abstützung können ein kortiko-spongiöser Span, eine zusätzliche Platte oder ein Cage Verwendung finden.

Die ventrale Zugangstechnik kann über die klassischen Zugangswege, minimal invasiv mit entsprechenden Spreizern und endoskopisch assisiert erfolgen.

Die offene ventrale Spondylodese kann über eine postero-laterale Thorakotomie,
Abb. 4 Dorso-ventrales Vorgehen mit Platzierung eines Cage bei LWK-2-Fraktur. (a) Unfallbilder (CT und 3 D CT), (b) Röntgenverlauf 1 Jahr nach Unfall. laterale Thorakotomie erfolgen.

Die Operation wird in Seitenlage vorgenommen, dabei werden in Abhängigkeit von der Lage der Aorta Verletzungen zwischen Th9 und LWK4 von links angegangen, da hier die Vena cava, Aorta und Leber den Zugang von rechts schwieriger gestalten. Entgegen der sonst üblichen Praxis in der Thoraxchirurgie wird auf eine Aufklappung verzichtet, um keine Fehlstellung der Wirbelsäule zu induzieren $[3,24]$. Die Höhe des verletzten Segmentes wird unter Bildverstärker auf der Thoraxwand markiert, um die Thorakotomie möglichst klein halten $\mathrm{zu}$ Hautschnitt wird zunächst die Muskulatur der Thoraxwand durchtrennt und die darunter liegende Rippe aus dem Verbund der Interkostalmuskulatur unter Schonung des interkostalen Gefäßnervenbündels geschält. Nach der Resektion der Rippe werden in den offenen Thorax ein Rippenspreizer sowie Haken für die Lunge bzw. das Zwerchfell eingesetzt. Im Bereich des thorakolumbalen Überganges ist es meist erforderlich, den Zwerchfellansatz von der Wirbelsäule und den angrenzenden Rippen abzulösen, um die Segmente BWK 12 bis LWK 2 versorgen zu können. Der verletzte Wirbelsäulenabschnitt wird nochmals unter Bildverstärker lokalisiert.

Soweit es das Ausmaß der Zerstörung des Wirbelkörpers zulässt, kann die Teilkorporektomie bei monosegmentaler Fusion auf den kranialen Anteil des Wirbels und die angrenzende Bandscheibe beschränkt bleiben, um die Fusion möglichst kurzstreckig zu gestalten (Abb.4).

Bei bisegmentaler Fusion ist auch die Resektion der kaudalen Bandscheibe erfor-
Thorakolumbophrenotomie oder eine können. Über einen etwa $15 \mathrm{~cm}$ langen derlich. Die entstandene Knochenlücke wird ausgemessen und ein entsprechend langer trikortikaler Span vom Beckenkamm entnommen. Bei Patienten mit geringer Weichteildeckung wird der Beckenkamm mit einer 1/3-Rohr-Platte rekonstruiert [4]. Bei sehr kräftigem Beckenkamm kann der Span bikortikal entnommen und somit die Kontur erhalten werden. Der Span wird pressfit in den Wirbelkörper eingesetzt. Bei zusätzlicher dorsaler Instrumentierung belassen es einige Autoren bei der reinen Spaninterposition. Als Alternative wird ein Schrauben- und Plattenimplantat zur Sicherung des Spans und der Fusion oder ein Cage bis zum endgültigen knöchernen Durchbau eingesetzt $[3,12,18]$.

\section{Laterale Thorakotomie}

Gegenüber der Thorakolumbophrenotomie nach Louis [24] besitzt dieser Zugang eine geringere Morbidität und verkürzte Operationszeit mit wenig Blutverlust. Hierbei wird in Rechtsseitenlage über der 10. Rippe eine 10 bis $12 \mathrm{~cm}$ lange Inzision durchgeführt, die von der Verlängerung der hinteren Axillarlinie bis zum Rippenbogen liegt. Im hinteren Wundbereich muss der M. latissimus dorsi, im vorderen der M. obliquus abdominis externus teilweise durchtrennt werden. Nach Thorakotomie wird die Lunge mit einem feuchten Tuch bedeckt und mit einem Lungenspatel weggehalten. Die Pleura parietalis wird in Längsrichtung in der Mitte der seitlichen Wand der Wirbelkörper inzidiert. Das Diaphragma wird zwischen Crus laterale und Crus intermedium längs gespalten.

\section{Lumbotomie}

Läsionen der LWS unterhalb LWK 2 werden von links über eine Lumbotomie versorgt. Auch hier wird die Läsionshöhe unter Zuhilfenahme des Röntgenbildverstärkers angezeichnet und ein etwa $10 \mathrm{~cm}$ langer Hautschnitt vorgenommen. Nach der Durchtrennung von Haut, Subkutis, M. obliquus externus und Anteilen des M. quadratus lumborum wird der $M$. transversus abdominis in Längsfaserrichtung gespalten. Unmittelbar darunter findet sich der Peritonealsack, der nach ventral/medial stumpf vom M. quadratus lumborum und M. iliopsoas abgeschoben wird. Der M. iliopsoas wird über der Wirbelsäule in Längsfaserrichtung gespalten und nach ventral und dorsal abgeschoben um auf die Segmentgefäße und die knöchernen Strukturen eingehen zu können. 


\section{Häufigkeitsverteilung der einzelnen Verfahren}

2/3 aller operativen Eingriffe am thorakolumbalen Übergang werden von dorsalen durchgeführt.

In der Untersuchungsserie der Deutschen Gesellschaft für Unfallchirurgie an 18 unfallchirurgischen Kliniken wurden 682 Patienten mit Frakturen des thoracolumbalen Überganges prospektiv erfasst. 448 (65,7\%) Patienten wurden von dorsal, $197(28,9 \%)$ kombiniert von dorsoventral und $37(5,4)$ isoliert von ventral operiert. $72 \%$ der 448 von dorsal stabilisierten Patienten erhielten eine transpedikuläre Spongiosaplastik. Die kombiniert dorsoventralen Eingriffe wurden bei $75(38,1 \%)$ Verletzten einzeitig und bei $122(61,9 \%)$ zweizeitig durchgeführt. Die durchschnittliche Operationszeit betrug bei kombiniertem Eingriff 4 Stunden, 14 Minuten. Es ließ sich eine statistisch signifikante Korrelation zwischen dem Ausmaß der neurologischen Beeinträchtigung und der Einengung des Spinalkanals im präoperativen CT nachweisen [23].

\section{Schlussfolgerung}

1. Frakturen im thorakolumbalen Übergang stellen mit über $50 \%$ die Hauptlokalisation der Verletzungen der Wirbelsäule dar.

2. Es gibt eindeutige Korrelationen zwischen der Einengung des Spinalkanals und neurologischen Ausfallserscheinungen. Ebenso nimmt die neurologische Beeinträchtigung vom Frakturtyp A zum Frakturtyp $\mathrm{C}$ zu.

3. Evidence based Aussagen zum Nachweis einer Verbesserung der neurologischen Befunde durch den operativen Eingriff lassen sich zur Zeit noch nicht erstellen.

4. Die funktionellen Ergebnisse nach operativer Stabilisierung im Brustund Lendenwirbelbereich sind den konservativen Verfahren überlegen.

5. Zur dorsalen Instrumentierung werden heute nur noch winkelstabile Implantate z.B. das USS angewandt.

6. Die ventrale Abstützung und Dekompression lässt sich über die klassischen Zugänge nach Louis, laterale Thorakotomien und mit Hilfe spezieller Spreizinstrumentarien sowie endoskopisch assistiert durchführen. Entscheidend für die Ergebnisse ist der Erfahrungsschatz des Operateurs.
7. Die ventrale Abstützung kann durch Span, zusätzliche Plattenfixateursysteme und durch Cages erreicht werden.

8. Aussagen über die Wertigkeit der dorsalen, ventralen und kombinierten Verfahren im Hinblick auf Spätresultate können zur Zeit noch nicht erbracht werden.

\section{Literatur}

${ }^{1}$ Bastian L, Knop C, Lange U, Blauth M. Transpediculäre Implantation von Schrauben in den thorakolumbalen Wirbel. Ergebnisse von Methoden, Häufigkeit und Komplikationen. Orthopädie 1999; 8: 693-702

2 Bedbrook GM. Spinal injuries with tetraplegia and paraplegia. J Bone Joint Surg (Br) 1979; 61: 267-284

${ }^{3}$ Beisse R. Destruktive Veränderungen. Die Wirbelsäule, Herausgeber A. v. Strempel. Georg Thieme Verlag, Stuttgart 2001; $198-240$

${ }^{4}$ Blauth M, Knop C, Bastian L, Lobenhoffer P. Neue Entwicklungen in der Chirurgie der verletzten Wirbelsäule. Orthopädie 1997; 26: $437-449$

${ }^{5}$ Blauth M, Knop C, Bastian L. Brust- und Lendenwirbelsäule. In: Tscherne Unfallchirurgie Wirbelsäule. Springer-Verlag Heidelberg Berlin, New York, Tokyo 1998

${ }^{6}$ Böhler L. Die Technik der Knochenbruchbehandlung. Maudrich, Wien 1951

7 Boerger TO, Limb D, Dickson RA. Does „Canal Clearance“ Affect Neurological Outcome After Thoracolumbar Burst Fractures? J Bone Joint Surg (Br) 2000; 5: 629-635

${ }^{8}$ Bradford DS, McBride GG. Surgical management of thoracolumbar spine fractures with incomplete neurologic deficits. Clin Orthop 1987; 218: 201 - 216

${ }^{9}$ Cusick JF, Myklebust JB, Ayvoloski M, Sances Jr. A, Houterman A, Larson SJ. Effects of vertebral column distraction in the monkey. J Neurosurg 1982; 57: 651 - 659

${ }^{10}$ Daniaux H. Transpedikuläre Reposition und Spongiosaplastik bei Wirbelkörperbrüchen der unteren Brust- und Lendenwirbelsäule. Unfallchirurg 1986; 89: 197-213

${ }^{11}$ Denis F, Armstrong GW, Searls K, Matta L. Acute thoracolumbar burst fractures in the absence of neurologic deficit: a comparison between operative and non-operative treatment. Clin Orthop 1984; 189: $142-149$

12 Dunn HK. Anterior stabilization of thoracolumbar injuries. Clin Orthop 1984; 189: $116-124$

${ }^{13}$ Gaines RW. The Use of Pedicle-Screw Internal fixation for the Operative Treatment of Spinal Disorders. J Bone Joint Surg 2000; 82 A: $1458-1476$

${ }^{14}$ George DC, Krag MH, Johnson CC, van Hal ME, Haugh LD, Grobler LJ. Hole preparation techniques for transpedicle screws. Effect on pull-out strength from human cadaveric vertebrae. Spine 1991; 16: $181-184$

${ }^{15}$ Gertzbein SD. Scoliosis Research Society: multicenter spine fracture study. Spine 1992; 17: $528-540$

${ }^{16}$ Hashimoto T, Kaneda K, Abumi K. Relationship between traumatic spinal instability other than scoliosis. S Afr J Surg 1967; 5: 7

17 Junge A, Gotzen L, v. Garrel T, Ziring E, Giannadakis K. Die monosegmentale Fixateur interne-Instrumentation und Fusion in der Be- handlung von Frakturen der thorakolumbalen Wirbelsäule. Unfallchirurg 1997; 100: $880-887$

${ }^{18}$ Kaneda K, Abumi K, Fujiya M. Burst fractures with neurologic deficits of the thoracolumbar-lumbar spine. Results of anterior decompression and stabilization with anterior instrumentation. Spine 1984: 9: $788-795$

${ }^{19}$ Kawaguchi Y, Matsui H, Tsuji H (1994) Back muscle injury after posterior lumbar spine surgery. Part 2: histologic and histochemical analyses in humans. Spine 19: 2598-2602

${ }^{20} \mathrm{Kim}$ NH, Lee HM, Chun IM. Neurologic Injury and Recovery in Patients With Burst Fracture of the Thoracolumbar Spine. Spine 1999; 24 3: $290-294$

${ }^{21}$ Knop C, Blauth M, Bastian L, Lange U, Becker T, Tscherne H. Frakturen der thorakolumbalen Wirbelsäule - Spätergebnisse nach dorsaler Instrumentierung und ihre Konsequenzen. Unfallchirurg 1997; 100: 630-639

${ }^{22}$ Knop C, Blauth M, Bühren V, Hax P-M, Kinzl L, Mutschler W, Pommer A, Ulrich C, Wagner S, Weckbach A, Wentzensen A, Wörsdörfer O. Operative Behandlung von Verletzungen des thorakolumbalen Übergangs, Teil 1: Epidemiologie. Unfallchirurg 1999; 102: $924-$ 935

${ }^{23}$ Knop C, Blauth M, Bühren V, Hax P-M, Kinzl L, Mutschler W, Pommer A, Ulrich C, Wagner S, Weckbach A, Wentzensen A, Wörsdörfer O. Operative Behandlung von Verletzungen des thorakolumbalen Übergangs, Teil 2: Operation und röntgenologische Befunde. Unfallchirurg 2000; 103: 1032 - 1047

${ }^{24}$ Louis R. Die Chirurgie der Wirbelsäule. Springer-Verlag Berlin, Heidelberg, New York, Tokyo (1985)

${ }^{25}$ Meyer PR, Heim S. Fractures of the thoracic spine T1 - T10. In: Meyer PR (ed) Surgery of spine trauma. Churchill Livingstone, Edinburgh 1989; $525-572$

${ }^{26}$ Mumford J, Weinstein JN, Spratt KF, Goel VK. Thoracolumbar burst fractures. The clinical efficacy and outcomes of nonoperative management. Spine 1993; 18: 955 -970

${ }^{27}$ Pedowitz RA, Garfin SR, Massie JB et al. Effects of magnitude and duration of compression on spinal nerve root conduction. Spine 1992; 17: 194-199

${ }^{28}$ Roy-Camille R, Saillant G, Mazel C. Internal fixation of the lumbar spine with pedicle screw plating. Clin Orthop 1986; 203: 7-17

\section{Prof. Dr. med. Hans-Jörg Oestern} Chefarzt

Allgemeines Krankenhaus Celle Klinik für Unfall- und Wiederherstellungschirurgie Siemensplatz 4 D-29223 Celle 